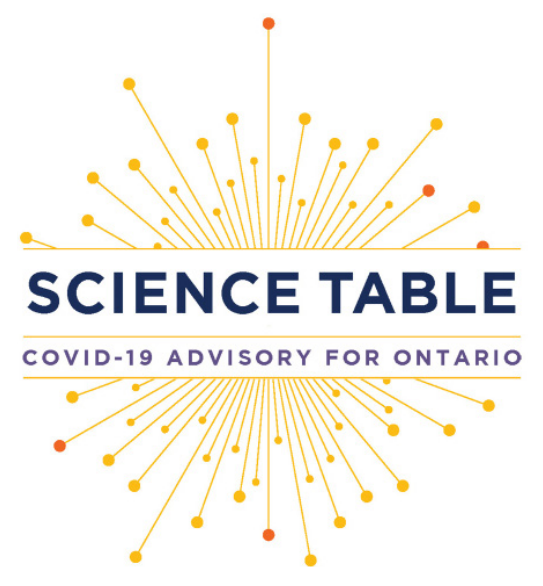

Version: $\mathbf{1 . 0}$

Published: September 3, 2021

Citation: Carlin S, Morris AM, Abdurrahman Z, et al. Heparin anticoagulation for hospitalized patients with COVID-19. Science Briefs of the Ontario COVID-19 Science Advisory Table. 2021;2(41). https://doi.org/10.47326/ ocsat.2021.02.41.1.0

Author Affiliations: The affiliations of the members of the Ontario COVID-19 Science Advisory Table can be found at https:// covid19-sciencetable.ca/.

Declarations of Interest: The declarations of interest of the members of the Ontario COVID-19 Science Advisory Table, its Working Groups, or its partners can be found at https:// covid19-sciencetable.ca/. The declarations of interest of external authors can be found under additional resources at https://doi. org/10.47326/ocsat.2021.02.41.1.0

About Us: The Ontario COVID-19 Science Advisory Table is a group of scientific experts and health system leaders who evaluate and report on emerging evidence relevant to the COVID-19 pandemic, to inform Ontario's response. Our mandate is to provide weekly summaries of relevant scientific evidence for the COVID-19 Health Coordination Table of the Province of Ontario, integrating information from existing scientific tables, Ontario's universities and agencies, and the best global evidence. The Science Table summarizes its findings for the Health Coordination Table and the public in Science Briefs.

The Drugs \& Biologics Clinical Practice Guidelines Working Group is a group of clinicians and scientists with recognized expertise in drugs, biologics, and clinical care. The Working Group evaluates existing scientific data, disease epidemiology, drug availability, and implementation issues in order to develop Clinical Practice Guidelines for the treatment of COVID-19 using drugs and biologics. The Working Group reports its findings to the public and the Science Table. Its findings are also summarized in Science Briefs.

Correspondence to: Secretariat of the Ontario COVID-19 Science Advisory Table (info@covid19-sciencetable.ca)
SCIENCE BRIEFS

\section{Heparin Anticoagulation for Hospitalized Patients with COVID-19}

Stephanie Carlin, Andrew M. Morris, Zainab B. Abdurrahman, Jacob J. Bailey, Martin E. Betts, William Ciccotelli, Bradley J. Langford, Elizabeth Leung, Antonina Maltsev, Laveena Munshi, Ullanda Niel, Nathan M. Stall, Anupama Wadhwa, Peter Jüni, Menaka Pai, on behalf of the Ontario COVID-19 Science Advisory Table and the Drugs \& Biologics Clinical Practice Guidelines Working Group

\section{Key Message}

\section{Critically III Patients}

Prophylactic dose low molecular weight or unfractionated heparin are recommended in critically ill patients hospitalized with COVID-19. These patients should not receive therapeutic dose anticoagulation unless they have a separate indication for this treatment. Therapeutic dose anticoagulation in this patient population does not reduce the need for organ support and may increase bleeding events as compared to prophylactic dose anticoagulation.

\section{Moderately III Patients}

Therapeutic dose low molecular weight or unfractionated heparin may be considered over prophylactic dose anticoagulation in moderately ill patients who are felt to be at low risk of bleeding. All other patients should receive prophylactic dose anticoagulation, unless they have a separate indication for therapeutic dose anticoagulation. Therapeutic dose anticoagulation may reduce the need for organ support (including the need for high-flow nasal oxygen) and appears to decrease thrombotic events in moderately ill patients compared to lower intensity anticoagulation. Its benefits on survival are unclear, and it may increase major bleeding events. Given the small absolute risk reduction for patient-important outcomes and the known harms, a strong recommendation for therapeutic dose anticoagulation in moderately ill patients cannot be made.

\section{Mildly III Patients}

There is insufficient evidence to make a recommendation around anticoagulation for mildly ill patients.

\section{Lay Summary}

Patients with COVID-19 may become ill because of a combination of viral infection and the inflammation produced by the body's immune system in response to that infection. Inflammation can lead to blood clots which damage their lungs, heart, kidney, and other vital organs.

\section{How Low Molecular Weight and Unfractionated Heparin Work}

Unfractionated heparin is a blood thinner (also called an anticoagulant). Low molecular weight heparins (including drugs like dalteparin, enoxaparin and tinzaparin) are also anticoagulants, which come from unfractionated heparin that has been broken down into smaller pieces. These anticoagulant medications make it more difficult for blood to clot and can be given in different doses to prevent clots (prophylactic dose) or to 
Copyright: 2021 Ontario COVID-19 Science Advisory Table. This is an open access document distributed under the terms of the Creative Commons Attribution License, which permits unrestricted use, distribution, and reproduction in any medium, provided that the original work is properly cited.

The views and findings expressed in this Science Brief are those of the authors and do not necessarily reflect the views of all of the members of the Ontario COVID-19 Science Advisory Table, its Working Groups, and its partners. treat clots (therapeutic dose). Additionally, there is some evidence from laboratory experiments not conducted in humans that unfractionated and low molecular weight heparins may reduce inflammation. Other laboratory experiments have shown that unfractionated heparin may prevent the SARS-CoV-2 virus from attacking cells.

\section{How We Came to Our Recommendations}

To understand low molecular weight and unfractionated heparin's effects on patients admitted to hospital with COVID-19, we reviewed a multiplatform randomized controlled trial (RCT) - a study that coordinated three similar RCTs to provide information on a larger group of patients - and a multicentre RCT. The multiplatform and multicentre RCTs compared the effects of these medications for treatment (therapeutic doses) and prevention (prophylactic doses). These studies involved thousands of patients in many different countries. They looked at patients with moderate illness (who were on low flow oxygen) as well as patients with severe illness (who required breathing and/or circulation support, including high flow oxygen).

\section{Our Recommendation for Patients Admitted to Hospital with COVID-19 with Critical Illness}

Patients admitted to hospital with severe COVID-19 illness do not benefit from treatment dose anticoagulation, and may suffer harm from severe bleeding or the need for a blood transfusion. They should not receive therapeutic dose anticoagulation unless they need it for another medical condition. Prevention dose anticoagulation should be offered to these patients.

\section{Our Recommendation for Patients Admitted to Hospital with COVID-19 with Moderate Illness}

Therapeutic dose anticoagulation may be given to patients admitted to hospital with moderate COVID-19 illness who are felt to be at a low risk of bleeding, after a discussion between the patient and the treating physician. This treatment may reduce the number of days that these patients spend receiving "intensive care", including high doses of oxygen, a ventilator, and/or medications that increase their blood pressure or help their heart pump. Therapeutic dose anticoagulation may reduce mortality, but may also increase the chance of severe bleeding or the need for a blood transfusion. For these reasons, therapeutic dose anticoagulation may not be appropriate for all patients. Prevention dose anticoagulation should be offered to all patients not on therapeutic dose, to reduce the chance that they will develop blood clots.

\section{Our Recommendation for Patients with COVID-19 with Mild IIIness}

We do not have enough evidence to make a recommendation about anticoagulation for patients with mild COVID-19 illness, who are usually not admitted to hospital. In general, these patients do not receive anticoagulation unless they need it for another medical condition.

\section{Side Effects of Anticoagulation}

The most common side effect of anticoagulant medications is bleeding. Higher doses of anticoagulants are more likely to cause bleeding. In moderately ill patients admitted to hospital with COVID-19 who have a low risk of bleeding, the benefits of therapeutic dose anticoagulation may outweigh the risk of bleeding. Other patients may receive lower, preventative doses of anticoagulation.

In critically ill patients admitted to hospital with COVID-19, the benefits of therapeutic dose anticoagulation do not outweigh the risks of bleeding. These patients do not benefit from a higher dose of anticoagulant treatment. They should receive lower, prevention doses of anticoagulation. 


\section{Background}

COVID-19-associated thrombosis, provoked by profound inflammation and viralmediated effects on cells, has been reported since the early days of the pandemic. ${ }^{1}$ Despite the use of prophylactic dose anticoagulation, venous and arterial thrombosesincluding in situ pulmonary thrombosis, deep vein thrombosis, pulmonary embolism, myocardial infarction, stroke, intravenous catheter thrombosis, and limb ischemiaare common in patients with COVID-19. A meta-analysis of studies utilizing various thromboprophylactic strategies reported venous thromboembolism in 5.5\% of admitted non-critically ill patients and $18.7 \%$ of critically ill patients when patients were not screened for venous thrombosis with imaging, and rates of $23 \%$ and $45.6 \%$ in noncritically ill and critically ill patients respectively when routine screening was used. ${ }^{2}$

Unfractionated and low molecular weight heparins are commonly used parenteral anticoagulants for the prevention and treatment of venous and arterial thromboembolism. Unfractionated heparin is a mixture of proteoglycans of various chain lengths which bind to anti-thrombin to inhibit coagulation factors. Low molecular weight heparins, including dalteparin, enoxaparin, and tinzaparin, are smaller chain length molecules fractionated from heparin which also bind to anti-thrombin and inhibit coagulation factors Xa and Ila.

In addition to their anticoagulant activity, heparins have anti-inflammatory and, possibly, antiviral activity. Anti-inflammatory effects occur through inhibition of neutrophil activation and function, interaction with vascular endothelium to prevent inflammatory mediator expression, prevention of vascular smooth muscle cell proliferation, ${ }^{3}$ and anticoagulant activity. In vitro studies have demonstrated that unfractionated heparin may bind to the SARS-CoV- 2 spike protein and act as a competitive inhibitor for viral entry into the host cell. ${ }^{4}$ As such, it was hypothesized that an increased heparin dose in COVID-19 patients may improve outcomes through multiple mechanisms.

\section{Questions}

Does anticoagulation improve outcomes, such as mortality, need for mechanical ventilation, and organ support-free days for patients with COVID-19?

What are the indications for therapeutic dose and prophylactic dose heparin in moderately and critically ill patients?

What is the risk of major bleeding in moderately and critically ill patients?

\section{Findings}

\section{Overview of Key Trials}

\section{Multiplatform Trial Including ATTACC, ACTIV-4a and REMAP-CAP}

The randomized multiplatform trial including ATTACC, ACTIV-4a and REMAP-CAP compared therapeutic anticoagulation with usual care thromboprophylaxis (standard prophylactic or intermediate dose) in hospitalized COVID-19 patients. Enrollment was discontinued in the critically ill cohort on December 19, 2020, after an interim analysis showed that the statistical criterion for futility had been met. On January 22, 2021, enrollment in the non-critically ill cohort was discontinued on the advice of the data and safety monitoring boards after a planned analysis of data in accordance with the adaptive design. Papers describing both arms were published on August 4, 2021. ${ }^{5,6}$

The multiplatform RCT used a composite primary outcome combining in-hospital mortality and days free of organ support. This outcome was an ordinal scale combining in-hospital mortality (assigned a value of -1) and days free of organ support (invasive 
or non-invasive mechanical ventilation, high flow nasal oxygen, vasopressor therapy, or extracorporeal membrane oxygenation (ECMO)) to day 21 (worst score of -1 and best score of 21). For example, a patient who died at day 18 would be assigned a score of -1 and a patient who survived and no longer required organ support at day 15 would be assigned a score of 6 . The multiplatform RCT also considered other patientimportant outcomes such as survival to hospital discharge without thrombosis, bleeding, survival to hospital discharge, survival without organ support through 28 days, and survival without intubation; these were considered secondary outcomes.

The trial in the critically ill cohort enrolled 1,089 patients between April 21, 2020 and December 19, 2020 in 10 countries (United Kingdom, Ireland, Netherlands, Australia, New Zealand, Saudi Arabia, Canada, United States, Brazil and Mexico). The trial in the moderately ill cohort enrolled 2,219 patients between April 21, 2020 and January 22, 2021 in 9 countries (United States, Canada, United Kingdom, Brazil, Mexico, Nepal, Australia, Netherlands, and Spain). A substantial proportion of patients in this multiplatform trial did not receive other treatments with demonstrated evidence for benefit: only $60 \%$ of moderately ill patients were on corticosteroids (a therapy that is now nearly universally used in this population), $0.5 \%$ were on tocilizumab, and $36 \%$ were on remdesivir.

\section{RAPID Trial}

The randomized multicentre RAPID trial comparing therapeutic anticoagulation with thromboprophylaxis (prophylactic standard dose) in moderately ill hospitalized COVID-19 patients completed enrollment on April 12, 2021, and was published in preprint form on July $12,2021 .^{7}$

The RAPID trial's primary outcome was a composite of intensive care unit (ICU) admission, non-invasive (bilevel or continuous positive airway pressure) or invasive mechanical ventilation, or death up to 28 days. Secondary outcomes included: allcause death; the composite of any mechanical ventilation or all-cause death; ICU admission or all-cause death; ventilator-free days alive; organ support-free days alive; ICU-free days alive; hospital free days alive; renal replacement therapy; venous thromboembolism; arterial thromboembolism; and D-dimer level at 2 days \pm 24 hours post-randomization.

The trial included 465 patients enrolled between May 29, 2020 and April 12, 2021 at 28 sites in 6 countries in 10 countries (Canada, Saudi Arabia, Brazil, United States, Ireland and United Arab Emirates). Patients were required to have D-dimer levels above the upper limit of normal (ULN) of the local hospital in the presence of an oxygen saturation $\leq 93 \%$ on room air, or $\geq 2$ times the ULN irrespective of oxygen saturation. Compared to the multiplatform trial, more patients in the RAPID trial received other treatments with demonstrated evidence for benefit; $77 \%$ of patients were on steroids, $5 \%$ were on tocilizumab, and $13 \%$ were on remdesivir.

\section{Outcomes in Moderately III Patients}

The multiplatform trial showed that therapeutic-dose anticoagulation increased organ support-free days as compared to usual care thromboprophylaxis with a posterior probability of $99.0 \%$ (median adjusted odds ratio $1.29,95 \%$ credible interval (Crl) 1.04 to 1.61). A total of $76.4 \%$ of patients in the usual care thromboprophylaxis group survived to hospital discharge without requiring organ support during the first 21 days, compared with $80.2 \%$ in the therapeutic-dose group. The median adjusted absolute improvement in organ support-free survival to hospital discharge was $4.6 \%$ with therapeutic anticoagulation (95\% $\mathrm{Crl} 0.7$ to 8.1$)$. There was no statistically significant difference between groups in the secondary endpoint of survival to hospital discharge, with $91.8 \%$ of patients in the usual care thromboprophylaxis surviving to hospital discharge as compared to $92.7 \%$ in the therapeutic dose anticoagulation 
group (median odds ratio adjusted for age, sex, site, D-dimer stratum, and time epoch of enrollment 1.21, 95\% Crl 0.87-1.68).

The RAPID trial showed no difference in the primary outcome (a composite of ICU admission, non-invasive or invasive mechanical ventilation, or death up to 28 days) between groups. This outcome occurred in $16.2 \%$ assigned to therapeutic heparin and $21.9 \%$ assigned to prophylactic heparin (odds ratio, $0.69 ; 95 \%$ confidence interval [Cl], 0.43 to $1.10, p=0.12$ ). The therapeutic heparin group had a lower incidence of death; death from any cause occurred in 4 patients (1.8\%) with therapeutic heparin and in 18 (7.6\%) with prophylactic heparin (odds ratio, $0.22 ; 95 \%-\mathrm{Cl}, 0.07$ to 0.65 ).

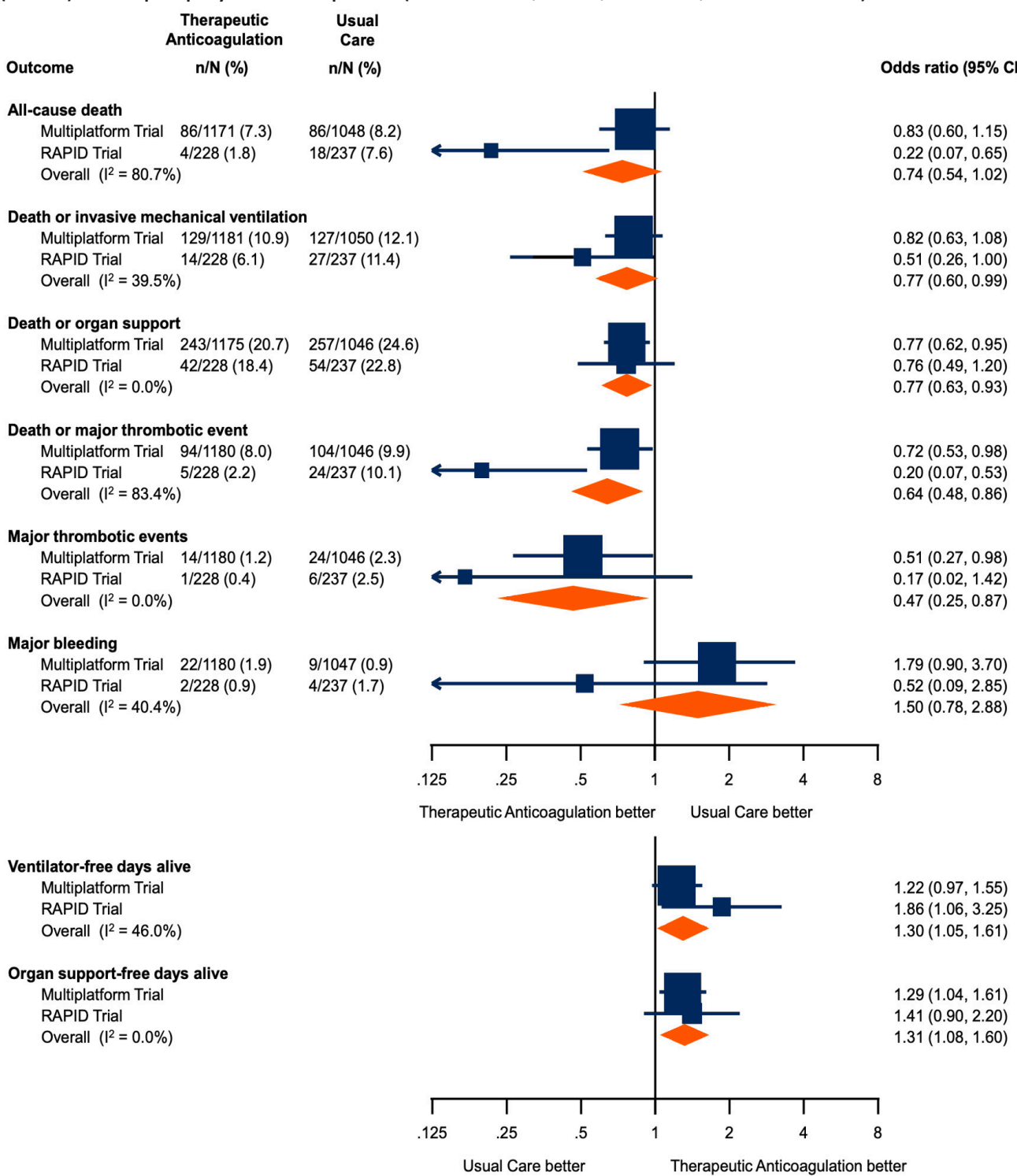

Figure 1. Meta-Analyses of the Effect and Safety of Anticoagulation Versus Usual Care in Moderately III Patients Forest plot presenting the odds ratios of efficacy and safety outcomes in moderately ill patients. Each square presents the results of an individual RCT with the size of squares proportional to the weights used in the meta-analysis and the horizontal lines indicating the $95 \% \mathrm{Cl}$. The solid vertical line at 1 represents the null effect (no effect on analyzed outcome) and the diamonds indicate the overall summary estimates from fixed-effect meta-analyses. Figure adapted from Sholzberg et al (2021). ${ }^{7}$ RCT, randomized controlled trial. Cl, confidence interval.

Major bleeding occurred in $1.86 \%$ in the therapeutic anticoagulation arm as compared to $0.86 \%$ in the usual care thromboprophylaxis arm in the multiplatform trial, with a median adjusted proportional odds ratio for the secondary endpoint of freedom from major bleeding with therapeutic anticoagulation as compared to usual care thromboprophylaxis of 0.56 (95\% Crl 0.27-1.11). Overt and symptomatic bleeding in a critical area or organ were rare with 9 events in the therapeutic anticoagulation 
group and 1 event in the usual care thromboprophylaxis group. There were 3 fatal bleeds in the therapeutic anticoagulation group and 1 fatal bleed in the usual care thromboprophylaxis group). Major bleeding occurred in 2 patients in the therapeutic heparin group and 4 patients in the prophylactic group (odds ratio $0.52 ; 95 \%-\mathrm{Cl}$, 0.09 to 2.85 ) in the RAPID trial. There were no fatal bleeding events and no cases of intracranial hemorrhage in this trial.

A meta-analysis of RAPID and the multiplatform was performed, to evaluate safety and efficacy outcomes for moderately ill patients. There was no significant reduction in all-cause death (odds ratio, $0.74 ; 95 \%-\mathrm{Cl}, 0.54$ to 1.02 ) with therapeutic heparin. There were significant reductions in the composite of death or invasive mechanical ventilation (odds ratio, $0.77 ; 95 \%-\mathrm{Cl}, 0.60$ to 0.99 ), death or organ support (odds ratio, $0.77 ; 95 \%-\mathrm{Cl}, 0.63$ to 0.93 ), death or major thrombotic event (odds ratio, $0.64 ; 95 \%-\mathrm{Cl}$, 0.48 to 0.86 ), and major thrombotic events (odds ratio, $0.47 ; 95 \%-\mathrm{Cl}, 0.25$ to 0.87 ). There were significant decreases in ventilator-free days alive (odds ratio, 1.30; 95\%$\mathrm{Cl} 1.05$ to 1.61) and organ support-free days alive (odds ratio 1.31, 95\% 1.08 to 1.60) with therapeutic heparin. There was a non-significant increase in major bleeding. ${ }^{7}$

\section{Outcomes in Critically III Patients}

The multiplatform trial demonstrated that the median adjusted proportional odds ratio for the effect of therapeutic anticoagulation on organ support-free days was 0.87 (95\% Crl $0.70-1.08$ ), yielding a posterior probability of futility of $99.8 \%$ and a posterior probability of inferiority of $89.4 \%$. The median value for organ supportfree days was 3 (interquartile range $-1,16$ ) in participants randomized to therapeutic anticoagulation as compared to 5 (interquartile range $-1,16$ ) in patients assigned to usual care thromboprophylaxis.

Major bleeding occurred in $3.1 \%$ of patients in the therapeutic anticoagulation group as compared to $2.4 \%$ of patients in the usual care thromboprophylaxis group in the multiplatform trial, with a median adjusted proportional odds ratio for the safety endpoint of major bleeding with therapeutic anticoagulation as compared to usual care thromboprophylaxis of 1.46 (95\% Crl 0.61-3.49).

\section{Practical Considerations}

Low molecular weight and unfractionated heparins are commonly used in the care of hospitalized patients. Protocols dealing with their use in specialized patient populations (e.g., renal failure, obesity) have been developed. In the Ontario context, we believe there are no specific concerns around cost, provincial supply, equity, feasibility, or acceptability associated with our recommendations.

Should Therapeutic Dose Anticoagulation Be Continued or Initiated in Critically III Patients Who Show Clinical Improvement, and Are Now Considered Moderately III?

We recommend continuation of prophylactic dose anticoagulation in critically ill patients who improve and can be classified as moderately ill, unless they develop another indication for therapeutic dose anticoagulation. This strategy was used in the multiplatform trial protocol, and is likely to be perceived as feasible and acceptable by clinicians. Moreover, the putative anti-inflammatory effects of therapeutic dose anticoagulation, potentially important to prevent progression to critical illness, are likely less important in patients who are clinically improving. At this time, there is insufficient evidence to support "stepping up" to therapeutic dose anticoagulation for patients who have clinically improved from critical to moderate illness.

Should Therapeutic Dose Anticoagulation Be Continued in Moderately III Patients Who Become Critically III? 
We recommend continuation of therapeutic dose anticoagulation in moderately ill patients felt to be at low risk of bleeding who become critically ill, unless they develop a contraindication. This strategy was used in the multiplatform trial protocol, and is likely to be perceived as feasible and acceptable by clinicians. At this time, there is insufficient evidence to support "stepping down" to prophylactic dose anticoagulation at the time of transfer to a critical care setting.

\section{Should We Use Standard or Intensified Prophylactic Dose Low Molecular Weight or Unfractionated Heparin?}

There is insufficient evidence at this time to recommend an intensified prophylactic dose of anticoagulation (also called "intermediate dose") in patients admitted to hospital with COVID-19. A multicentred RCT including 562 critically ill patients at 10 Iranian centres compared standard with intermediate dose anticoagulation (at a dose higher than standard prophylaxis, but lower than therapeutic anticoagulation) found no difference with respect to the composite endpoint of venous or arterial thrombosis, treatment with ECMO or 30 day mortality. Very low rates of venous thromboembolism were reported, however, and the study was underpowered. ${ }^{14}$

\section{Should We Use Low Molecular Weight Heparin or Unfractionated Heparin?}

Given the greatervariability in anticoagulant response, increased laboratory monitoring requirements, and higher incidence of heparin induced thrombocytopenia with unfractionated heparin as compared to low molecular weight heparin, low molecular weight heparin is generally preferred over unfractionated heparin. ${ }^{12}$ Intravenous unfractionated heparin may, however, be considered in patients with severe renal dysfunction (creatinine clearance less than $30 \mathrm{~mL} / \mathrm{min}$ ), who would benefit from a shorter acting anticoagulant (e.g., those with high bleeding risk, those who require brief periods of anticoagulant interruption), or in situations where subcutaneous medication absorption may be reduced or erratic (i.e., anasarca).

\section{Special Considerations with Pregnancy}

While pregnant patients were excluded from the ACTIV-4a and RAPID trials (pregnancy not specified among exclusion criteria in ATTACC and REMAP-CAP trials), pregnant patients are at increased risk of morbidity and mortality due to COVID-19. ${ }^{15}$ Low molecular weight and unfractionated heparins, due to their molecular size, do not cross the placenta. ${ }^{16}$ Given the extensive experience and safety associated with these anticoagulants in pregnancy, we believe that the recommendations outlined in this document should apply to pregnant patients.

\section{Special Considerations with Breastfeeding}

Unfractionated heparin, due to its molecular size, is not found in breastmilk. Small amounts of low molecular weight heparin may pass into breast milk, however, given their low bioavailability, do not result in an anticoagulant effect in the baby. Given the extensive experience and safety associated with both of these anticoagulants in lactation, we believe that the recommendations outlined in this document should apply to lactating patients.

\section{Recommendations}

Please see the below section entitled "Methods Used for this Scientific Brief" for a description of COVID-19 illness severity criteria.

\section{Critically III Patients}

Prophylactic dose low molecular weight or unfractionated heparin are recommended in critically ill patients hospitalized with COVID-19. These patients should not receive 
therapeutic dose anticoagulation unless they have a separate indication for this treatment. Therapeutic dose anticoagulation in this patient population does not reduce the need for organ support and may increase bleeding events as compared to prophylactic dose anticoagulation.

The panel did not specifically review the indirect evidence for the use of prophylactic dose anticoagulation versus no anticoagulation in critically ill patients with COVID-19. Risk assessment models to estimate thrombotic risk in critically ill patients exist, but none have been specifically validated in patients with COVID-19. ${ }^{8,9}$ However critically ill patients with COVID-19 are at higher risk of venous thromboembolism by virtue of having an acute infectious disease, organ failure, and may be considered to have a hypercoagulable state (given their high incidence of clinically evident thrombosis). Many critically ill patients also have additional risk factors for thrombosis, including older age and immobility. For this reason, all critically ill patients are considered high risk for thrombosis, even after routine prophylactic anticoagulation; pharmacologic venous thromboembolism prophylaxis is accepted as standard of care, unless there are clear bleeding contraindications. ${ }^{10,11}$ For this reason, prophylactic dose anticoagulation is likely to be perceived as feasible and acceptable by clinicians and patients, versus no anticoagulation. The rates of major bleeding in critically ill patients on prophylactic dose anticoagulation is approximately $5 \%$; this is comparable to the findings of the multiplatform trial. ${ }^{12}$

\section{Moderately III Patients}

Therapeutic dose low molecular weight or unfractionated heparin may be considered over prophylactic dose anticoagulation in moderately ill patients who are felt to be at low risk of bleeding. All other patients should receive prophylactic dose anticoagulation, unless they have a separate indication for therapeutic dose anticoagulation. Therapeutic dose anticoagulation may reduce the need for organ support (including the need for high-flow nasal oxygen) and appears to decrease thrombotic events in moderately ill patients compared to lower intensity anticoagulation. Its benefits on survival are unclear, and it may increase major bleeding events. Given the small absolute risk reduction for patient-important outcomes and the known harms, a strong recommendation for therapeutic dose anticoagulation in moderately ill patients cannot be made at this time.

Risk assessment models to estimate bleeding risk in hospitalized patients exist, but none have been specifically validated in patients with COVID-19. ${ }^{13}$ The cumulative incidence of major in-hospital bleeding within 14 days of admission in acutely ill hospitalized medical patients is approximately $1.2 \%$. The panel defined patients at higher risk of bleeding as those who would have been excluded from the multiplatform trial: intracranial surgery or stroke within 3 months, history of intracerebral arteriovenous malformation, cerebral aneurysm or mass lesion of the central nervous system, intracranial malignancy, history of bleeding diatheses such as hemophilia, history of gastrointestinal bleeding within 3 months, thrombolysis within 1 week, epidural or spinal catheter, major surgery within 2 weeks, uncontrolled hypertension with systolic blood pressure over $200 \mathrm{mmHg}$ or diastolic blood pressure over $120 \mathrm{mmHg}$, platelet count less than $50 \times 10^{\wedge} 9 / \mathrm{L}$, INR over 2 , baseline aPTT over 50 seconds, hemoglobin less than $80 \mathrm{~g} / \mathrm{L}$, acute or subacute bacterial endocarditis, or concurrent use of dual antiplatelet therapy.

A minority $(1 / 3)$ of the guideline panel voted against the use of therapeutic dose anticoagulation versus prophylactic dose anticoagulation in moderately ill patients at low risk of bleeding. The majority $(2 / 3)$ of the guideline panel voted for a conditional recommendation for therapeutic dose anticoagulation versus prophylactic dose anticoagulation in moderately ill patients at low risk of bleeding. The panel's ultimate recommendation is based on the perceived importance of both mortality and organ 
support free days to patients (in particular, avoiding intubation and possible transfer to another institution if a higher level of care is needed). There was a small absolute benefit for these outcomes, and the panel noted that The RAPID trial suggested that though therapeutic dose anticoagulation may reduce all-cause death, this benefit was not sustained when data from the RAPID trial and the multiplatform trial were metaanalyzed. They also noted that both the multiplatform trial's and the RAPID trial's definition of organ support free days included avoiding respiratory support via highflow nasal cannula, an outcome perceived to be less important to many patients. The panel's ultimate recommendation is also based on the perceived importance of survival to hospital discharge to patients, an outcome that showed minimal absolute benefit in the multiplatform trial.

It should be noted that the available evidence for anticoagulation reflects patients who largely did not receive other evidence-based therapies for COVID-19 such as tocilizumab, and remdesivir. The panel hypothesized that with improved, evidencebased therapy, the small absolute benefits of anticoagulation may be less pronounced, while its bleeding risks would remain the same. The panel also notes that the benefits of therapeutic anticoagulation may have been diluted in the multiplatform trial, and the harms of prophylactic anticoagulation may have been overstated, as $26.5 \%$ (227/855) of patients in the usual care group received intermediate dose thromboprophylaxis.

The panel did not specifically review the indirect evidence for the use of prophylactic dose anticoagulation versus no anticoagulation in moderately ill patients with COVID-19. Risk assessment models to estimate thrombotic risk in hospitalized patients exist, but none have been specifically validated in patients with COVID-19.8,9 Patients with COVID-19 are at higher risk of venous thromboembolism by virtue of having an acute infectious disease, and may be considered to have a hypercoagulable state (given their high incidence of clinically evident thrombosis). Many moderately ill patients also have additional risk factors for thrombosis, including age, immobility, and respiratory failure. For this reason, prophylactic dose anticoagulation is likely to be perceived as feasible and acceptable by clinicians and patients, versus no anticoagulation.

\section{Mildly III Patients}

There is currently insufficient evidence to make a recommendation around anticoagulation for mildly ill patients.

\section{Methods Used for This Science Brief}

We searched PubMed, Google Scholar, the COVID-19 Rapid Evidence Reviews, the Joanna Briggs Institute's COVID-19 Special Collection, LitCovid in PubMed, the Oxford COVID-19 Evidence Service, the World Health Organization's Global Literature on Coronavirus Disease, and other COVID-19 specific resources listed by the Guidelines International Network and the McMaster Health Forum. In addition, we retrieved reports citing relevant articles through Google Scholar and reviewed references from identified articles for additional studies. The search was last updated on August 20, 2021.

For therapeutic recommendations, we used the following definitions for severity:

\section{Critically III}

Patients requiring ventilatory and/or circulatory support, including high-flow nasal oxygen, non-invasive ventilation, invasive mechanical ventilation, or ECMO. These patients are usually managed in an intensive care setting.

Moderately III 
Patients newly requiring low-flow supplemental oxygen. These patients are usually managed in hospital wards.

Mildly III

Patients who do not require new or additional supplemental oxygen from their baseline status, intravenous fluids, or other physiological support. These patients are usually managed in an ambulatory/outpatient setting.

\section{Author Contributions}

SC wrote the first draft of the Science Brief. All authors contributed to the conception of the Science Brief, revised it critically for important intellectual content, and approved the final version.

The authors would like to thank all members of the Drugs and Biologics Clinical Practice Guideline Working Group for their contribution to this Science Brief. Members are listed here: https://covid19-sciencetable.ca/about/.

The Ontario COVID Drugs and Biologics Clinical Practice Guidelines and associated Science Briefs are currently available at https://antimicrobialstewardship.com and https://covid19-sciencetable.ca/sciencebrief/.

\section{Acknowledgements}

The authors would like to acknowledge the important contributions of the Ontario COVID-19 Antimicrobial and Immunomodulatory Clinical Practice Guidelines Committee throughout the COVID-19 pandemic. Those members include: Karim Ali, MD, Niagara Health System; Amir Amiri, Patient Partner; Nisha Andany, MD MPH, Sunnybrook Health Sciences Centre; Sally Bean, Ethicist, Sunnybrook Health Sciences Centre; Zain Chagla, MD, Hamilton Health Sciences; Bill Ciccotelli, MD, Grand River Hospital ; Pavani Das, MD, North York General Hospital; Linda Dresser, PharmD, University Health Network; Sameer Elsayed, MD, LHSC Victoria Hospital; Wayne Gold, MD, University Health Network; Kevin Gough, MD, St. Michael's Hospital, Unity Health Toronto; Chris Graham, MD, Trillium Health Partners; Rebecca Greenberg, Ethicist, Sinai Health; Shahid Husain, MD MS, University Health Network; Neal Irfan, PharmD BScPhm, Hamilton Health Sciences; Susan John, MD, Scarborough Health Network; Rupert Kaul, MD PhD, University Health Network; Elizabeth Leung, PharmD MSCl, St. Michael's Hospital, Unity Health Toronto; Brian Minnema, MD, St. Joseph's Hospital, Unity Health Toronto; Jeya Nadarajah, MD, MSc, Markham Stouffville Hospital; Caroline Nott, MD, The Ottawa Hospital; Lesley Palmay, BSc BScPhm MSc, Sunnybrook Health Sciences Centre; Alexandra Persichino, BScPhm, Thunder Bay Regional Health Sciences Center; Sumit Raybardhan, MPH, North York General Hospital; Kathryn Timberlake, PharmD, Hospital for Sick Children; Liliana Volnikova, Patient Partner; Anupma Wadhwa, MD MEd, Hospital for Sick Children; Evan Wilson, MD MSc, Kingston Health Sciences Centre; Peter Wu, MD MSc, University Health Network; Ivan Ying, MD, Mackenzie Health.

\section{References}

1. Gupta A, Madhavan MV, Sehgal K, et al. Extrapulmonary manifestations of COVID-19. Nat Med. 2020;26(7):1017-1032. https://doi.org/10.1038/s41591020-0968-3

2. Nopp S, Moik F, Jilma B, Pabinger I, Ay C. Risk of venous thromboembolism in patients with COVID-19: A systematic review and meta-analysis. Res Pract Thromb Haemost. 2020;4(7):1178-1191. https://doi.org/10.1002/rth2.12439 
3. Poterucha TJ, Libby P, Goldhaber SZ. More than an anticoagulant: Do heparins have direct anti-inflammatory effects? Thromb Haemost. 2017;117(03):437-444. https://doi.org/10.1160/TH16-08-0620

4. Hippensteel JA, LaRiviere WB, Colbert JF, Langouët-Astrié CJ, Schmidt EP. Heparin as a therapy for COVID-19: current evidence and future possibilities. Am J PhysiolLung Cell Mol Physiol. 2020;319(2). https://doi.org/10.1152/ajplung.00199.2020

5. ATTACC, ACTIV-4a, and REMAP-CAP Investigators. Therapeutic anticoagulation with heparin in critically ill patients with Covid-19. N Eng/ J Med. 2021;(0). https:// doi.org/10.1056/NEJMoa2103417

6. ATTACC, ACTIV-4a, and REMAP-CAP Investigators. Therapeutic anticoagulation with heparin in noncritically ill patients with Covid-19. N Engl J Med. Published online August 4, 2021. https://doi.org/10.1056/NEJMoa2105911

7. Sholzberg $\mathrm{M}$, Tang $\mathrm{GH}$, Rahhal $\mathrm{H}$, et al. Heparin for moderately ill patients with Covid-19. Published online July 9, 2021:2021.07.08.21259351. https://doi.org/10 .1101/2021.07.08.21259351

8. Barbar S, Noventa F, Rossetto V, et al. A risk assessment model for the identification of hospitalized medical patients at risk for venous thromboembolism: the Padua Prediction Score. J Thromb Haemost. 2010;8(11):2450-2457. https://doi. org/10.1111/j.1538-7836.2010.04044.x

9. Nendaz M, Spirk D, Kucher N, et al. Multicentre validation of the Geneva Risk Score for hospitalised medical patients at risk of venous thromboembolism. Explicit ASsessment of Thromboembolic RIsk and Prophylaxis for Medical PATients in SwitzErland (ESTIMATE). Thromb Haemost. 2014;112(03):531-538. https://doi. org/10.1160/TH13-05-0427

10. Alhazzani W, Lim W, Jaeschke RZ, Murad MH, Cade J, Cook DJ. Heparin thromboprophylaxis in medical-surgical critically ill patients: a systematic review and meta-analysis of randomized trials. Crit Care Med. 2013;41(9):2088-2098. https://doi.org/10.1097/CCM.0b013e31828cf104

11. Cook D, Crowther M, Meade M, et al. Deep venous thrombosis in medical-surgical critically ill patients: prevalence, incidence, and risk factors. Crit Care Med. 2005;33(7):1565-1571. https://doi.org/10.1097/01.CCM.0000171207.95319.B2

12. The PROTECT Investigators for the Canadian Critical Care Trials Group and the Australian and New Zealand Intensive Care Society Clinical Trials Group. Dalteparin versus unfractionated heparin in critically ill patients. N Engl J Med. Published online April 7, 2011. https://doi.org/10.1056/NEJMoa1014475

13. Decousus H, Tapson VF, Bergmann J-F, et al. Factors at admission associated with bleeding risk in medical patients: Findings from the IMPROVE investigators. CHEST. 2011;139(1):69-79. https://doi.org/10.1378/chest.09-3081

14. INSPIRATION Investigators, Sadeghipour P, Talasaz AH, et al. Effect of intermediatedose vs dtandard-dose prophylactic anticoagulation on thrombotic events, Extracorporeal Membrane Oxygenation treatment, or mortality among patients with COVID-19 admitted to the intensive care unit: the INSPIRATION randomized clinical trial. JAMA. Published online March 18, 2021. https://doi.org/10.1001/ jama.2021.4152

15. Zambrano LD. Update: Characteristics of symptomatic women of reproductive age with laboratory-confirmed SARS-CoV-2 infection by pregnancy status - United States, January 22-October 3, 2020. MMWR Morb Mortal Wkly Rep. 2020;69. https://doi.org/10.15585/mmwr.mm6944e3

16. American College of Obstetricians and Gynecologists. ACOG practice bulletin No. 
196: Thromboembolism in pregnancy. Obstet Gynecol. 2018;132(1). https://doi. org/10.1097/AOG.0000000000002706 